\title{
Sodium-Glucose Cotransporter 2 Inhibitors in the Treatment of Type 2 Diabetes
}

\author{
Nikolaos Papanas* and Theano Penlioglou \\ Second Department of Internal Medicine, Democritus University of Thrace, University Hospital of Alexandroupolis, Greece
}

Received: 眥 June 20, 2018; Published: 酬 June 27, 2018

*Corresponding author: Nikolaos Papanas, Diabetes Centre, Second Department of Internal Medicine, Democritus University of Thrace, University Hospital of Alexandroupolis, G Kondyli 22c, Alexandroupolis 68100, Greece

\section{Editorial}

Over the past years, type 2 diabetes mellitus (T2DM) has become a global pandemic, both in developed and developing countries $[1,2]$. Its aetiology is multifactorial, including genetic factors, increasing age, obesity and insulin resistance [3]. Among these factors, obesity is of paramount importance, and its management can be of benefit $[4,5]$.

Consequently, the ideal anti diabetic medication should promote weight loss or at least prevent further weight increase [6]. Sodiumglucose cotransporter 2 inhibitors (SGLT-2is), i.e. dapagliflozin, empagliflozin, canagliflozin and others, represent a new class of agents approved for the management of T2DM, which promote weight loss [7]. Indeed, SGLT-2is promote glycosuria, thereby leading to: a) reduction of serum glucose; b) loss of calories through the urine [7-9]. Normally, around $90 \%$ of filtered renal glucose is reabsorbed in the first segment of the proximal tubule by SGLT- 2 . These agents prevent renal glucose re absorption by inhibition of SGLT-2 [7-9]. Interestingly, canagliflozin has an additional mode of action: at $300 \mathrm{mg}$, but not at $150 \mathrm{mg}$, it also reduces intestinal glucose absorption during the meal following drug administration [10].

By virtue of their mode of action, SGLT-2is are not dependent on insulin secretion and/or resistance $[7,11]$. Thus, they can be combined with any other glucose lowering agent, including insulin $[7,11]$. Moreover, they can even be used as a monotherapy in patients unable to tolerate metformin [7]. The mean HbA1c reduction with these agents is $-0.66 \%$ vs. placebo and $-0.06 \%$ vs. active comparator [12]. The mean weight loss is $-1.8 \mathrm{~kg}$ [12]. Nowadays, there is evidence that SGLT- 2 inhibitors may prove a therapeutic option even for patients with type 1 diabetes mellitus in the future, reducing weight and insulin dosage $[13,14]$.

A very important advantage of SGLT-2is is that they do not cause hypoglycaemias $[7,11,12]$. This is because they do not act via insulin secretion. However, caution is needed when they are combined with sulfonylureas. Indeed, dose of the latter may need to be reduced to avoid hypoglycaemias, because a substantial amount of serum glucose is reduced via secretion in the urine $[7,11,12]$.

Of particular relevance, SGLT-2is harbour numerous other beneficial actions [11]. Most importantly, empagliflozin and canagliflozin have been demonstrate to significantly and markedly reduce the composite endpoint of cardiovascular death, non-fatal myocardial infarction and non-fatal stroke, as well as hospitalisations for heart failure in randomised clinical trials including high-risk patients $[15,16]$. The mechanisms of these impressive beneficial actions relate to weight loss, reduction of blood volume, increase in haematocrit, sodium loss, reduced arterial calcification and others $[17,18]$. However, the exact role of these mechanisms remains to be elucidated $[17,18]$. The results of a cardiovascular trial with dapagliflozin are soon expected [19].

Empagliflozin, canagliflozin and dapagliflozin have also been evaluated in a large multinational registry of data from new users of these agents vs. other antidiabetic agents [20-22]. To adjust for differences in patient characteristics favouring or not SGLT-2i use, a meticulous propensity score matching was employed [20$22]$. The majority of patients received dapagliflozin in Europe and canagliflozin in the USA $[20,21]$. Impressively, reductions in total mortality, heart failure, cardiovascular deaths and major adverse cardiovascular events were seen [20,21]. A separate analysis of dapagliflozin is also available now [22]. Again, reductions of major adverse cardiovascular events, hospitalisation for heart failure, and all-cause mortality were observed with this agent [22].

Other beneficial actions of SGLT-2is include reduction of blood pressure [13] and serum uric acid [23], as well as improved serum lipids (reduction of triglycerides and increase of highdensity lipoprotein cholesterol) [13]. Moreover, they have been shown to reduce fatty liver infiltration $[24,25]$. The main proposed mechanisms of reduced blood pressure include osmotic diuresis, 
mild natriuresis, weight loss, and possible nitric oxide release [26]. Of note, SGLT-2is have also been demonstrated to preserve estimated glomerular filtration rate (eGFR) and to reduce albuminuria and kidney damage $[16,27,28]$.

What about untoward effects? The commonest are urogenital mycotic infections, most commonly in women [12,29]. Volume depletion, dehydration and hypotension might, theoretically, occur, but only in very old and frail patients [12,29]. Ketone bodies may be increased [30]. On very rare occasions, diabetic ketoacidosis without marked hyperglycaemia has been noted, but this is almost exclusively in insulinopenic or acutely ill patients, or in the setting of surgery and/or insulin withdrawal [15,31]. Canagliflozinassociated fractures and lower-extremity amputations have been seen, while the underlying mechanism remains elusive [16].

Of note, SGLT-2is should not be initiated in patients with an $(\mathrm{eGFR})<60 \mathrm{ml} / \mathrm{min}[7,11,12]$. In such patients, the anti diabetic effect is negligible. If a patient's eGFR is reduced from above 60 to $45-60 \mathrm{ml} / \mathrm{min}$, then dose reductions are possible for empagliflozin and canagliflozin, but not for dapagliflozin [7,11,12].

In conclusion, SGLT-2is are very useful antidiabetic agents. Not only do they improve glycaemic control, but they also reduce body weight and blood pressure and harbour other beneficial properties $[11,32,33]$. Most importantly, they reduce cardiovascular morbidity. Thus, they are being increasingly used and they appear to have a very bright future in the management of T2DM [11].

\section{Conflicts of Interest}

This editorial was written independently. The authors did not receive financial or professional help with the preparation of the manuscript. NP has been an advisory board member of TrigoCare International, Abbott, AstraZeneca, Elpen, MSD, Novartis, Novo Nordisk, Sanofi-Aventis and Takeda; has participated in sponsored studies by Eli Lilly, MSD, Novo Nordisk, Novartis and Sanofi-Aventis; received honoraria as a speaker for AstraZeneca, Boehringer Ingelheim, Eli Lilly, Elpen, Galenica, MSD, Mylan, Novartis, Novo Nordisk, Pfizer, Sanofi-Aventis, Takeda and Vianex; and attended conferences sponsored by TrigoCare International, AstraZeneca, Boehringer Ingelheim, Eli Lilly, Novartis, Novo Nordisk, Pfizer and Sanofi-Aventis.

\section{References}

1. International Diabetes Federation (2017) IDF Diabetes Atlas, $8^{\text {th }}$ Edition.

2. Hu FB, Satija A, Manson JE (2015) Curbing the Diabetes Pandemic: The need of global policy solutions. JAMA 313(23): 2319-2320.

3. Tamayo T, Rosenbauer J, Wild SH, et al. (2014) Diabetes in Europe: An update. Diabetes Res Clin Pract 103(2): 206-217.

4. Grams J, Garvey WT (2015) Weight loss and the prevention and treatment of type 2 diabetes using lifestyle therapy, pharmacotherapy, and bariatric surgery: mechanisms of action. Curr Obes Rep 4(2): 287302.

5. Eckel RH, Kahn SE, Ferrannini E, et al. (2011) Obesity and type 2 diabetes: what can be unified and what needs to be individualised? J Clin Endocrinol Metab 96(6): 1654-1663.
6. Papanas N, Maltezos E (2012) Antidiabetic treatment: Though lovers be lost, love shall not. World J Diabetes 3(9): 158-160.

7. Nauck MA (2014) Update on developments with SGLT2 inhibitors in the management of type 2 diabetes. Drug Des Devel Ther 8: 1335-1380.

8. Davies M, Chatterjee S, Khunti K (2016) The treatment of type 2 diabetes in the presence of renal impairment: what we should know about newer therapies. Clin Pharmacol 8: 61-81.

9. Tsimihodimos V, Filippatos TD, Elisaf MS (2018) SGLT2 inhibitors and the kidney: Effects and mechanisms. Diabetes Metab Syndr.

10. Stein P, Berg JK, Morrow L, et al. (2014) Canagliflozin, a sodium glucose co-transporter 2 inhibitor, reduces post-meal glucose excursion in patients with type 2 diabetes by a non-renal mechanism: results of a randomised trial. Metabolism 63(10): 1296-1303.

11. Pafili K, Papanas N (2015) Luseogliflozin and other sodiumglucose cotransporter 2 inhibitors: no enemy but time? Expert Opin Pharmacother 16(4): 453-456.

12. Vasilakou D, Karagiannis T, Athanasiadou E, et al. (2013) Sodium-glucose cotransporter 2 inhibitors for type 2 diabetes: a systematic review and meta-analysis. Ann Intern Med 159(4): 262-274.

13. Mittermayer F, Caveney E, De Oliveira C, et al. (2017) Addressing unmet medical needs in type 1 diabetes: a review of drugs under development. Curr Diabetes Rev 13(3): 300-314.

14. Pafili K, Maltezos E, Papanas N (2017) Dapagliflozin for the treatment of type 1 diabetes mellitus. Expert Opin Investig Drugs 26(7): 873-881.

15.Zinman B, Wanner C, Lachin JM, et al. (2015) Empagliflozin, cardiovascular outcomes, and mortality in type 2 diabetes. N Engl J Med 373(22): 2117-2128.

16. Neal B, Perkovic V, Mahaffey KW, et al. (2017) Canagliflozin and cardiovascular and renal events in type 2 diabetes. $N$ Engl J Med 377(7):644-657.

17. Papanas N, Tsioufis C, Maltezos ES (2016) Editorial: The New Trial EMPAREG-OUTCOMES in Type 2 Diabetes: "and Death Shall Have no Dominion"? Curr Vasc Pharmacol 14(4): 316-318.

18. Tsioufis C, Andrikou E, Thomopoulos C, Papanas N, Tousoulis D (2017) Oral glucose-lowering drugs and cardiovascular outcomes: from the negative RECORD and ACCORD to neutral TECOS and promising EMPAREG. Curr Vasc Pharmacol 15(5): 457-468.

19. Raz I, Mosenzon O, Bonaca MP, et al. (2018) DECLARE-TIMI 58: Participants' baseline characteristics. Diabetes Obes Metab 20(5): 11021110.

20. Kosiborod M, Cavender MA, Fu AZ, et al. (2017) Lower risk of heart failure and death in patients initiated on sodium-glucose cotransporter-2 inhibitors versus other glucose-lowering drugs: The CVD-REAL Study (Comparative Effectiveness of Cardiovascular Outcomes in New Users of Sodium-Glucose Cotransporter-2 Inhibitors). Circulation 136(3): 249259.

21. Birkeland KI, Jørgensen ME, Carstensen B, et al. (2017) Cardiovascular mortality and morbidity in patients with type 2 diabetes following initiation of sodium-glucose co-transporter-2 inhibitors versus other glucose-lowering drugs (CVD-REAL Nordic): a multinational observational analysis. Lancet Diabetes Endocrinol 5(9): 709-717.

22. Persson F, Nyström T, Jørgensen ME, et al. (2018) Dapagliflozin is associated with lower risk of cardiovascular events and all-cause mortality in people with type 2 diabetes (CVD-REAL Nordic) when compared with dipeptidyl peptidase-4 inhibitor therapy: A multinational observational study. Diabetes Obes Metab 20(2): 344-351.

23. Katsiki N, Papanas N, Mikhailidis DP (2010) Dapagliflozin: more than just another oral glucose-lowering agent? Expert Opin Investig Drugs 19(12): 1581-1589. 
24. Pafili K, Maltezos E, Papanas N (2018) Ipragliflozin and sodium glucose transporter 2 inhibitors to reduce liver fat: will the prize we sought be won? Expert Opin Pharmacother 19(3): 185-187.

25. Lee PCH, Gu Y, Yeung MY, et al. (2018) Dapagliflozin and empagliflozin ameliorate hepatic dysfunction among chinese subjects with diabetes in part through glycemic improvement: a single-center, retrospective, observational study. Diabetes Ther 9(1): 285-295.

26. Majewski C, Bakris GL (2015) Blood pressure reduction: an added benefit of sodium-glucose cotransporter 2 inhibitors in patients with type 2 diabetes. Diabetes Care 38(3): 429-430.

27. Wanner C, Inzucchi SE, Lachin JM, et al. (2016) Empagliflozin and progression of kidney disease in type 2 diabetes. N Engl J Med 375(18): 323-334.

28. Heerspink HJ, Heerspink HJ, Johnsson E, Gause-Nilsson I, Cain VA, et al. (2016) Dapagliflozin reduces albuminuria in patients with diabetes and hypertension receiving renin-angiotensin blockers. Diabetes Obes Metab 18(6): 590-597.
29. Halimi S, Vergès B (2014) Adverse effects and safety of SGLT-2 inhibitors. Diabetes Metab 40(6 Suppl 1): S28-34.

30. Pafili K, Maltezos E, Papanas N (2016) Pharmacokinetic and pharmacodynamic drug evaluation of tofogliflozin for the treatment of type 2 diabetes. Expert Opin Drug Metab Toxicol 8: 1-14.

31. Meyer EJ, Gabb G, Jesudason D (2018) SGLT2 Inhibitor-associated euglycemic diabetic ketoacidosis: a South Australian clinical case series and Australian spontaneous adverse event notifications. Diabetes Care 41(4): e47-e49.

32. Storgaard H, Gluud LL, Bennett C, et al. (2016) Benefits and harms of sodium-glucose co-transporter 2 inhibitors in patients with type 2 diabetes: a systematic review and meta-analysis. PLoS One 11(11): e0166125.

33. Ghosh RK, Bandyopadhyay D, Hajra A, Biswas M, Gupta A (2016) Cardiovascular outcomes of sodium-glucose cotransporter 2 inhibitors: A comprehensive review of clinical and preclinical studies. Int J Cardiol 212: $29-36$.

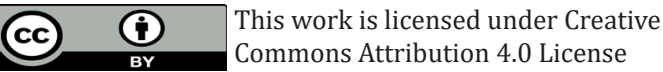

To Submit Your Article Click Here:

Submit Article

DOI: 10.32474/AD0.2018.01.000111

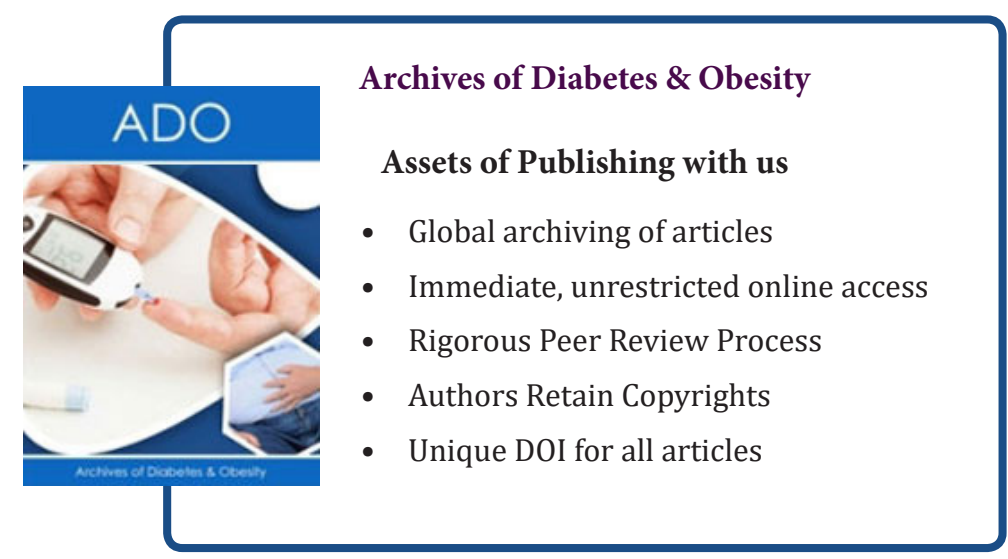

